\title{
Analysis of cancer-related mutations in extracellular vesicles RNA by Droplet Digital'M PCR
}

Soo Ann Yap ${ }^{1}$ iD , Agnieszka Münster-Wandowski ${ }^{2}$, Anika Nonnenmacher ${ }^{1}$, Ulrich Keilholz*,1,3 \& Sandra Liebs ${ }^{1,3}$

${ }^{1}$ Charité Universitätsmedizin Berlin, corporate member of Freie Universität Berlin, Humboldt-Universität zu Berlin \& Berlin Institute of Health, Charité Comprehensive Cancer Center, Berlin, Germany; ${ }^{2}$ Institute of Integrative Neuroanatomy, Charité Universitätsmedizin Berlin, corporate member of Freie Universität Berlin, HumboldtUniversität zu Berlin \& Berlin Institute of Health, Berlin, Germany; ${ }^{3}$ German Cancer Consortium (DKTK), German Cancer Research Center (DKFZ), Heidelberg, Germany; *Author for correspondence: ulrich.keilholz@charite.de

BioTechniques 69: 99-107 (August 2020) 10.2144/btn-2020-0028

First draft submitted: 11 March 2020; Accepted for publication: 7 May 2020; Published online: 25 June 2020

\section{ABSTRACT}

Extracellular vesicles (EVs) are taking their place as potential biomarkers in the field of liquid biopsy. In this study, EVs were isolated from plasma samples of 31 patients with colorectal cancer and melanoma via differential centrifugation and Droplet Digital ${ }^{T M}$ PCR (Bio-Rad, CA, USA) was used to profile BRAF V600E/K, KRAS G12A/C/D/V and KRAS G13D mutations from EV-derived cDNA. The concordance rates with corresponding tissue were $54 \%$ and $44 \%$ in the colorectal cancer and melanoma cohort, respectively. Two patients displayed mutations in EVs not previously detected in tissue as evidence for emerging molecular resistance to anti-EGFR and BRAF/MEK inhibitor therapy prior to radiological evidence of tumor progression. We concluded that EV-derived nucleic acids may provide clinically relevant diagnostic information and mirror evolution of the disease.

\section{METHOD SUMMARY}

Extracellular vesicles (EVs) derived from patient plasma were isolated via serial centrifugation, followed by EV-RNA isolation and EV-cDNA amplification. Amplified EV-cDNAs were analyzed with the Bio-Rad QX200TM ddPCR system (CA, USA). EVs were also morphologically visualized with transmission electron microscopy, while their proteomic content was verified by western blotting.

KEYWORDS:

colorectal cancer $\bullet$ ddPCR • exosomes • extracellular vesicles • liquid biopsy • melanoma

Liquid biopsy was introduced as a noninvasive approach to assist the gold standard tissue biopsy in predicting treatment response, disease progression, prognosis and early cancer detection [1,2]. The analysis of solid tissue is spatially limited and consequently it only describes the section of the tumor that was probed. In contrast, liquid biopsy is increasingly helping us to understand the heterogeneous landscape of the tumor [3]. Consisting of circulating tumor cells, circulating cell-free DNA (cfDNA) and extracellular vesicles (EVs), liquid biopsy has been thoroughly studied to provide molecular features of various tumor types [4].

EVs are nanosized, cup-shaped vesicles that play a role in intercellular communication in both the physiological and the pathological environment. The vesicles are capable of preparing premetastatic niches by orchestrating the ideal environment before guiding metastatic cells to the destination [5]. EVs are formed by inward budding of the plasma membrane and are released into the bloodstream at the end of the endosomal recycling pathway, while carrying cargo from the cell of origin [6]. EVs are considered to be a suitable cancer biomarker due to their ability to protect their biological cargo (protein, lipid, nucleic acid) in the circulation [7]. Nucleic acid mutations in patient-derived EVs have been detected in studies of glioblastoma [8], pancreatic cancer [9,10], colorectal cancer [11], advanced pancreatic and biliary cancer [12].

In our study, the oncogenes KRAS and BRAF were analyzed with Droplet Digital ${ }^{\mathrm{TM}}$ PCR (ddPCR; Bio-Rad, CA, USA) to compare gene status in EV-derived cDNA (EV-CDNA) isolated from patients' plasma with that in corresponding tissues to understand concordance rate in 31 colorectal cancer (CRC) and melanoma (MM) patients.

\section{Materials \& methods}

\section{Patients}

A total of 13 patients with advanced CRC and 18 with MM were enrolled into our study from the Department of Medical Oncology and Hematology and the Skin Tumor Center of the Charité Medical University. Among the 31 samples, three patients were previously recruited for OncoTrack from the Charité and the Medical University Graz [13]. All individuals provided informed consent. The study was approved by ethics committees from both Charité University Medicine (EA1/069/11, EA4/090/08, EA4/063/13) and Medical University Graz (23015 ex 10/11). Patient demographics are summarized in Table 1. 
Table 1. Demographics, clinical characterization and therapy information of participants from (A) the colorectal cancer and (B) the melanoma cohort.

\begin{tabular}{|c|c|c|c|}
\hline (A) Colorectal cancer & & (B) Melanoma & \\
\hline Total patients $(n=13)$ & Number & Total patients $(n=18)$ & Number \\
\hline Gender: & & Gender & \\
\hline - Male & 11 & - Male & 12 \\
\hline - Female & 2 & - Female & 6 \\
\hline Age (years) & 59 (median) & Age (years) & 54 (median) \\
\hline Localization: & & Stage: & \\
\hline - Right-sided colon cancer & 5 & -1 & 2 \\
\hline - Left-sided colon cancer & 5 & -2 & 0 \\
\hline - Rectum & 3 & -3 & 3 \\
\hline AJCC: & & -4 & 13 \\
\hline-1 & 0 & BRAF MUT & 13 \\
\hline-2 & 0 & BRAF WT & 5 \\
\hline-3 & 0 & Therapy: & \\
\hline-4 & 13 & - Nivolumab & 2 \\
\hline KRAS MUT & 7 & - Nivolumab/ipilimumab & 7 \\
\hline KRAS WT & 6 & - MEK inhibitor & 2 \\
\hline Therapy: & & $-B R A F+M E K$ inhibitor & 7 \\
\hline - Cetuximab & 3 & & \\
\hline - FOLFOX + cetuximab & 3 & & \\
\hline - FOLFOX + bevacizumab & 6 & & \\
\hline - FOLFOX + bevacizumab + panitumumab & 1 & & \\
\hline
\end{tabular}

AJCC: American Joint Committee on Cancer staging system; CRC: Colorectal cancer; MM: Melanoma; MUT: Mutation; WT: Wild-type.

\section{Isolation of plasma-derived EVs}

Peripheral blood was collected in BD Vacutainer ${ }^{\circledR}$ EDTA tubes (BD, NJ, USA) and centrifuged at $1811 \times g$ for 7 min and $3061 \times g$ for 10 min at room temperature. Plasma was stored at $-80^{\circ} \mathrm{C}$ until further processing. Plasma $(1 \mathrm{ml})$ was thawed at room temperature to isolate EVs via ultracentrifugation, as shown in Supplementary Figure 1. This was conducted with the Sorvall Discovery 90 SE ultracentrifuge and T-1270 fixed angle titanium rotor (Thermo Scientific, MA, USA).

\section{Isolation of cell lines-derived EVs}

For assay optimization and as positive controls, EVs were isolated via ultracentrifugation from cell lines (SK-MEL-28, IGR-1, RPMI 8226, MIA PaCa-2, LS174T, SW480 and HCT 116) that express mutations of BRAFV600E/K, KRAS G12A/C/D/V and KRAS G13D. Initially, cell lines were validated by single nucleotide polymorphism profiling with Multiplexion $\mathrm{GmbH}$ (Friedrichshafen, Germany) and tested negative for mycoplasma using Promokine PCR Mycoplasma Test KIT I/C based on the manufacturer's instructions (PromoCell GmbH, Heidelberg, Germany). At $80 \%$ confluency, primary fetal bovine serum was removed by rinsing adherent cells twice with phosphate-buffered saline (PBS) and reculturing them with advanced DMEM or advanced RPMI (Thermo Fisher Scientific, MA, USA) without fetal bovine serum. The EV-rich supernatant was harvested after $48 \mathrm{~h}$ of incubation at $37^{\circ} \mathrm{C}$ and $5 \% \mathrm{CO}_{2}$. The remaining cells were pelleted at $2000 \times g$ for $20 \mathrm{~min}$ at $4^{\circ} \mathrm{C}$ and the supernatant was filtered with a $0.22-\mu \mathrm{m}$ cellulose acetate filter (Merck, Darmstadt, Germany) to remove larger particles. The supernatant was concentrated with a Centricon Plus-70 Filter (Merck) following the supplier's instructions and was then filtered again, followed by two cycles of ultracentrifugation as depicted in Supplementary Figure 1. Cell lines, their corresponding mutation status and culture conditions are listed in Supplementary Table 1.

\section{RNA isolation \& whole transcriptome amplification of extracellular vesicles}

After isolation of EVs, RNA was extracted using the miRCURY RNA Isolation Kit - Cell \& Plants (Exiqon, Denmark) for cell line-derived EVs and the miRCURY RNA Isolation Kit - Biofluids (Exiqon, Denmark) for EVs from plasma. Due to the discontinuation of both Exiqon kits, the miRNEASY micro kit (Qiagen, Hilden, Germany) was used as a replacement for $50 \%$ of the samples. EV-RNA was isolated according to manufacturer's instructions. DNase inhibitor (Thermo Fisher Scientific) was added to RNA samples to avoid possible DNA contamination. The samples were purified, concentrated with the RNA Clean \& Concentrator ${ }^{T M}-5$ kit (Zymo Research, CA, USA) and eluted in $10 \mu l$ nuclease-free, diethyl pyrocarbonate-treated water (Carl Roth, Karlsruhe, Germany) based on the manufacturer's instructions.

Whole transcriptome amplification was performed with the REPLI-g WTA kit (Qiagen) according to the supplier's instructions. The EV-derived RNA was converted into cDNA and amplified, resulting in a final volume of $19 \mu \mathrm{l}$ amplified cDNA. Samples were purified, 
concentrated with the RNA Clean \& Concentrator-5 kit (Zymo Research) and eluted in $30 \mu$ nuclease-free, diethyl pyrocarbonate-treated water (Carl Roth) based on the manufacturer's instructions.

\section{Droplet Digital polymerase chain reaction}

Primers and probes for ddPCR assays were designed using Primer blast [14]; $2 \mu$ cDNA and $18 \mu$ of total reaction volume - consisting of primers, wild-type and mutation probes and ddPCR Supermix for Probes (no dUTP) (Bio-Rad, CA, USA) - were converted into droplets with the QX200 ${ }^{T M}$ droplet generator (Bio-Rad). Droplet-partitioned samples were transferred to a 96-well plate and PCR was performed on the T-100 thermal cycler (Bio-Rad) with the following protocol: $95^{\circ} \mathrm{C}$ for $10 \mathrm{~min}$, followed by 40 cycles of $94^{\circ} \mathrm{C}$ for $30 \mathrm{~s}$ and $58-61^{\circ} \mathrm{C}$ for $1 \mathrm{~min}$, with a final step of $98^{\circ} \mathrm{C}$ for $10 \mathrm{~min}$ (annealing temperatures varied with the different mutation assays). Primer and probe sequences and PCR conditions are listed in Supplementary Table 2. Samples were analyzed as duplicates in the FAM (MUT) and HEX (WT) channels using the QX200 ${ }^{T M}$ Droplet Reader (Bio-Rad). Droplet fluorescence data were analyzed with QuantaSoft ${ }^{T M}$ version 1.7.4 (Bio-Rad) and detected events were expressed as copies/ $\mu$ (CPM). The results were then calculated to [15]:

$$
\mathrm{CPM}=\frac{\text { copies of target } \mathrm{x} \text { template volume }}{\text { volume analyzed of the duplicates }}
$$

For ddPCR optimization, cDNAs from cell lines with wild-type or mutation status in KRAS and BRAF were utilized. The finalized mutation assays were repeated in triplicate. Optimization steps for the ddPCR are described in Supplementary Figures 2 and 3.

The false-positive rate was evaluated using the number of unspecific events detected in the mutation channel when analyzing nontemplate controls and high and low concentrations of cell line-derived wild-type cDNA (500 and 100 CPM, respectively). The limit of detection was identified by analyzing mutant cDNA diluted in a background of constant wild-type cDNA.

Assay specificity was verified, demonstrating that probes did not bind to other mutations at the same nucleotide position. For all assays, the false-positive rate ranged from zero to one event and the limits of detection were $0.1 \%$. Prior to analysis of patient samples, EVs derived from cell lines were utilized to validate detection of mutations.

\section{Whole-mount immunoelectron microscopy}

EV-rich fractions were fixed and deposited on Formvar carbon-coated (Plano, Wetzlar, Germany), glow-discharged (MED 020, Leica) nickel grids. The grids were washed in PBS and incubated in a blocking buffer containing $1 \%$ bovine serum albumin (Sigma-Aldrich, Darmstadt, Germany) in PBS. This was followed by an exposure to primary antibody CD63 produced in mouse (Bio-Rad) for $1 \mathrm{~h}$ and secondary antibody goat antimouse IgG conjugated to 5-nm gold particles (Plano) for 30 min. Subsequently, grids were stained with $2 \%$ phosphotungstic acid (Sigma-Aldrich), $\mathrm{pH} 7.0$, for $10 \mathrm{~min}$ and incubated in a mixture of $2 \%$ methylcellulose and $4 \%$ uranyl acetate (Merck) for 10 min on ice. Stainless steel loops were used to remove grids, excess fluid was gently blotted on Whatman Grade 1 qualitative cellulose filter paper (Merck) and the grids were left to dry. Analysis was performed using a Zeiss transmission electron microscope 912 equipped with a digital camera (Proscan $2 \mathrm{~K}$, Slow-Scan CCD-Camera, Zeiss, Oberkochen, Germany) and image analysis was performed with iTEM software (Olympus). For the negative controls, primary antibodies were omitted.

\section{Western blot}

EV pellets were homogenized in RIPA lysis buffer (50 mM Tris/HCL, pH 7.2-7.4, $150 \mathrm{mM} \mathrm{NaCl,} \mathrm{0.1 \%} \mathrm{SDS,} \mathrm{1 \%} \mathrm{Na-Deoxycholate,} \mathrm{1 \%} \mathrm{Triton}$ X100), supplemented with Protease Inhibitor Cocktail (Thermo Scientific) and boiled at $95^{\circ} \mathrm{C} ; 20 \mu \mathrm{g}$ protein was loaded in $4-15 \%$ gradient Mini Protean ${ }^{\circledR}$ TGX ${ }^{T M}$ Precast Gels (Bio-Rad). Proteins were electrophoretically transferred to polyvinylidene difluoride membranes and blocked in $5 \%$ bovine serum albumin in TBST (0.1\% Tween-20). Membranes were incubated with antibodies against tetraspanins CD 9 (1:1000 dilution), CD81 (1:500 dilution) and cis-matrix Golgi marker GM 130 (1:1000 dilution) (System Bioscience, CA, USA). Protein bands were visualized via Li-Cor Odyssey Imaging System (Li-Cor, NE, USA). Western blot analysis was performed in duplicate.

\section{Results \& discussion}

\section{Characterization of extracellular vesicles}

The features of EVs were examined using whole-mount immunoelectron microscopy and were determined based on their size, morphology and immunoreactivity for proteins enriched in EVs. Using these criteria, CD63-positive EVs ranging from 10-100 nm, with morphologies varying from cup-shaped to round with an intact membrane and a central depression, were identified in EVs derived from both cell lines and patient samples, consistent with reports by Trajkovic, Raposo and Colombo [16-18]. Based on the methods mentioned by György [19] and Théry, Ostrowski and Segura [20], the typical morphology of EVs was confirmed by including $2 \%$ phosphotungstic acid and $4 \%$ uranyl acetate as controls. To confirm the endocytic origin of the EVs and to distinguish vesicles of interest, immune-labeling of anti-CD63 conjugated to 5-nm gold nanoparticles was applied based on a refined method previously described by Théry et al. [21]. The expression level of tetraspanins fluctuated between different types of EVs and the density of CD63 labeling between EV fractions or between areas within a given fraction was not homogeneous. Some EVs were labeled, but there also appeared to be a population of larger unlabeled EVs (>100 nm) (Figure 1). 


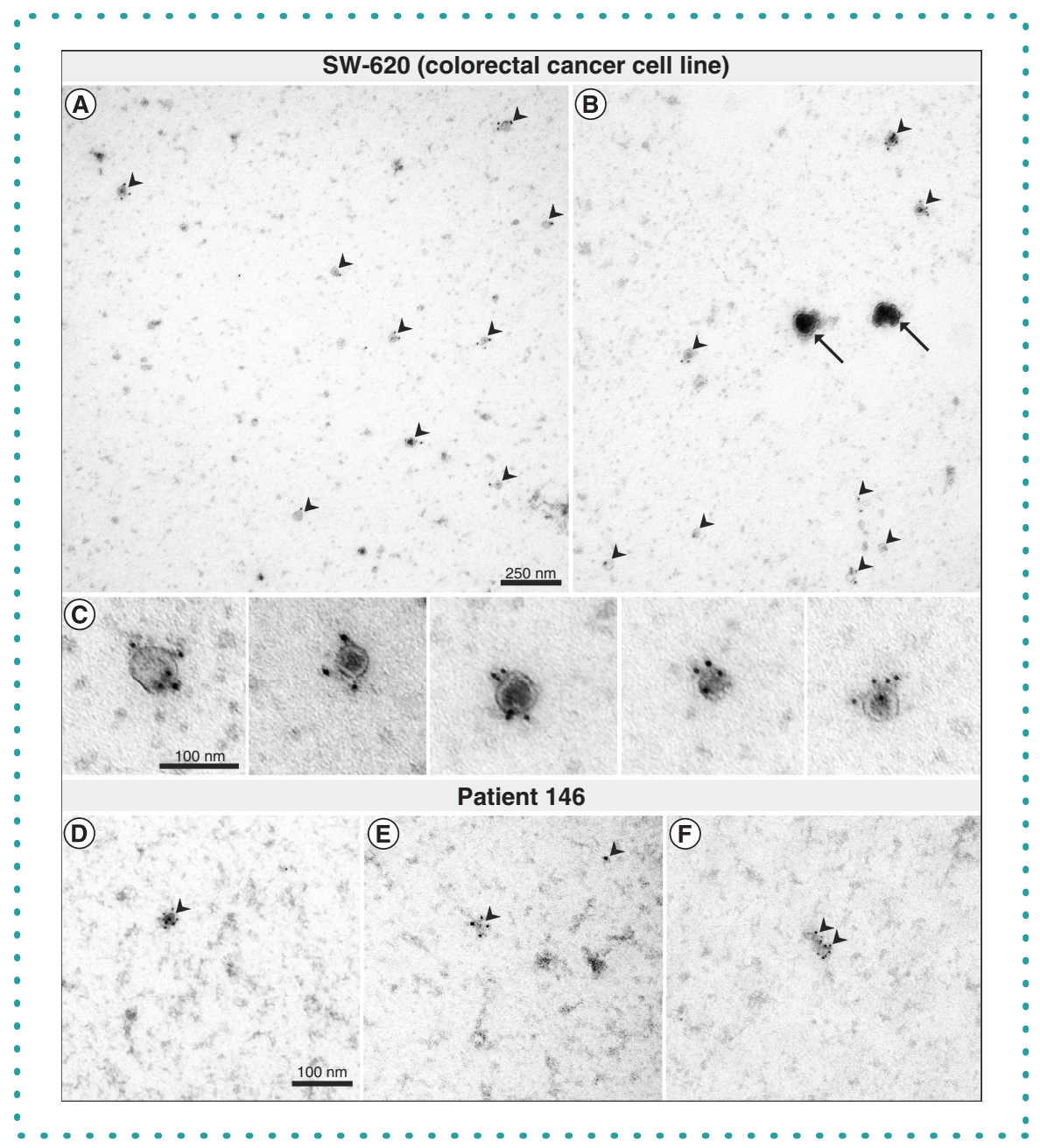

Figure 1. Immunoelectron microscopy images of whole-mount extracellular vesicles from colorectal cancer cell line and patient. (A \& B) Electron microscopy images of whole-mount extracellular vesicles from colorectal cancer cell line (SW-620) labeled for CD63. Arrowheads indicate CD63-positive extracellular vesicles (5-nm immunogold particles) and arrows demonstrate unlabeled microvesicles. (C) Inlets illustrate magnified images of extracellular vesicles. (D-F) Electron micrographs of whole-mount extracellular vesicles from patient 146 labeled for CD63.

For western blot analysis, tetraspanins CD9 and CD81 were detected in EVs isolated from cell lines and plasma samples. Due to the lack of a specific marker found ubiquitously in EVs, GM130 (a cis-matrix Golgi marker) was included as a negative control to label cell line-derived proteins which are not present in EVs (Figure 2).

\section{Mutation detection}

A total of $18 \mathrm{MM}$ and 13 advanced CRC patients with corresponding tissue samples were recruited in this study. Pretreatment tissue profiling revealed the presence of KRAS mutations in seven of $13 \mathrm{CRC}$ patients (54\%). BRAF was mutated in 13 of $18 \mathrm{MM}$ patients $(72 \%)$. $K R A S / B R A F$ wild-type was present in six CRC patients (46\%) and BRAF wild-type in $5 \mathrm{MM}$ patients (28\%). Thus EVs from MM patients were screened for BRAF V600E and BRAF V600K mutations, whereas EVs from CRC patients were screened for five KRAS mutations (KRAS G12A/C/D/V and KRAS G13D). In the MM cohort, BRAF wild-type was detected in ten EV samples, mutations were detected in six patients (V600E and V600K in two and four patients, respectively), and in two samples neither the wild-type nor any mutation was detected. In the CRC cohort, KRAS wild-type was detected in seven EV samples, mutations were present in three patients (G12D and $\mathrm{G} 12 \mathrm{~V}$ in two and one patient, respectively), and in three patients neither wild-type nor any mutation was detected.

EV-based ddPCR analysis was compared with tissues profiled during routine analysis (Tables $2 \& 3$ ). Among the $18 \mathrm{MM}$ patients, $44 \%(8 / 18)$ EV-derived results were in concordance with the gene status in tissue. BRAF wild-type instead of expected mutations was detected in $28 \%(5 / 18)$ of the patients and $17 \%(3 / 18)$ of the samples had mismatched mutations (V600K was detected twice instead of the expected V600E and V600E was detected once instead of wild-type). In CRC, 54\% (7/13) of EV-based profiles were in concordance with the respective tissue, whereas in $15 \%(2 / 13)$ of the samples wild-type was detected instead of the expected mutations (and vice versa for one patient). 


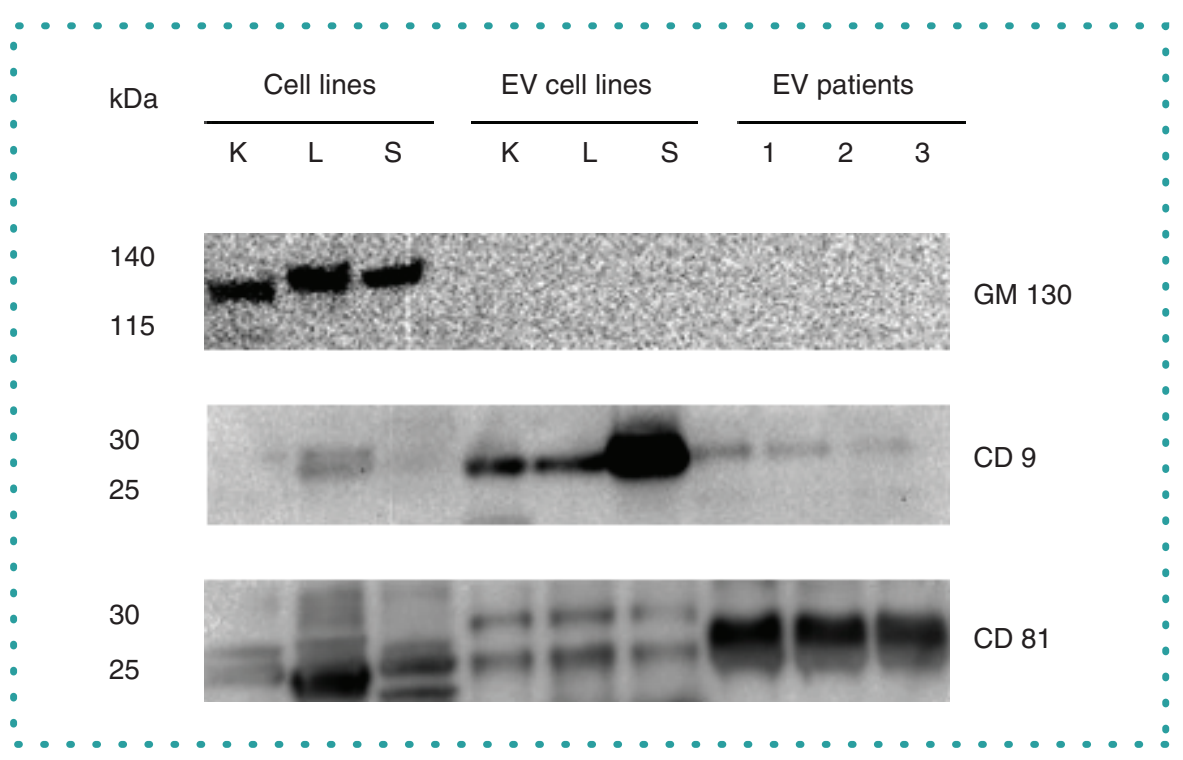

Figure 2. Characterization of extracellular vesicles by western immunoblotting. EVs derived from cell lines and plasma from three patients were analyzed by immunoblotting with antibodies against exosomal proteins CD9 and CD81 and nonexosomal protein, GM130. Whole cell lysates were included as positive control for GM130.

EV: Extracellular vesicle; K: Kelly, L: LS174T, S: SK-Mel-28.

Table 2. Concordance between gene status of extracellular vesicles and tumor tissue for (A) melanoma and (B) colorectal cancer cohort.

\begin{tabular}{|c|c|}
\hline \multicolumn{2}{|l|}{ (A) Melanoma } \\
\hline ddPCR results & Number of patients (\%) \\
\hline Total number of MM patients & 18 \\
\hline Concordance: & $8(44.4 \%)$ \\
\hline - BRAF WT & $5(27.7 \%)$ \\
\hline - BRAF V600E & $1(5.5 \%)$ \\
\hline - BRAF V600K & $2(11.2 \%)$ \\
\hline \multicolumn{2}{|l|}{ Discordance: } \\
\hline - BRAF WT instead of expected BRAF MUT & $5(27.7 \%)$ \\
\hline - BRAF V600K instead of expected BRAF V600E & $2(11.2 \%)$ \\
\hline - BRAF V600E instead of expected BRAF WT & $1(5.5 \%)$ \\
\hline Undetectable & $2(11.2 \%)$ \\
\hline \multicolumn{2}{|l|}{ (B) Colorectal cancer } \\
\hline ddPCR results & Number of patients (\%) \\
\hline Total number of CRC patients & 13 \\
\hline Concordance: & $7(53.9 \%)$ \\
\hline - KRAS WT & $5(38.5 \%)$ \\
\hline - KRAS G12D & $1(7.7 \%)$ \\
\hline - KRAS G12V & $1(7.7 \%)$ \\
\hline \multicolumn{2}{|l|}{ Discordance: } \\
\hline - KRAS WT instead of expected KRAS MUT & $2(15.4 \%)$ \\
\hline - KRAS MUT instead of expected KRAS WT & $1(7.7 \%)$ \\
\hline Undetectable & $3(23 \%)$ \\
\hline
\end{tabular}

CRC: Colorectal cancer; ddPCR: Droplet Digital ${ }^{T M}$ PCR; MM: Melanoma; MUT: Mutation; WT: Wild-type.

\section{Discordant cases of KRAS \& BRAF mutation status}

Two cases stood out with discordant mutation status between tissues and EVs. Patient 16 was diagnosed with melanoma and brain metastasis. Based on the BRAF V600E mutation status in cervical lymphatic nodules, the patient received dabrafenib, followed by dabrafenib and trametinib (BRAF/MEK inhibitor) combination therapy. The patient was recruited prior to receiving combination im- 


\begin{tabular}{|c|c|c|c|c|}
\hline \multicolumn{5}{|c|}{ (A) Colorectal cancer } \\
\hline Patient & Cancer stage (AJCC) & Tissue sequencing & ddPCR & CPM \\
\hline 6 & 4 & KRAS G12D & KRAS WT & 4.18 \\
\hline 10 & 4 & KRAS G12D & No results & No results \\
\hline 19 & 4 & KRAS G13D & No results & No results \\
\hline 21 & 4 & KRAS G12D & KRAS WT & 33.3 \\
\hline 22 & 4 & KRAS WT & KRAS WT & 1.87 \\
\hline 23 & 4 & KRAS WT & KRAS WT & 3.63 \\
\hline 25 & 4 & KRAS WT & KRAS WT & 8.58 \\
\hline 26 & 4 & KRAS WT & KRAS WT & 39.6 \\
\hline 27 & 4 & KRAS G13D & No results & No results \\
\hline 30 & 4 & KRAS WT & KRAS WT & 2508 \\
\hline 302 & 4 & KRAS G12D & KRAS G12D & 5.2 \\
\hline 338 & 4 & KRAS WT & KRAS G12D & 13.86 \\
\hline 356 & 4 & KRAS G12V & KRAS G12V & 3.5 \\
\hline \multicolumn{5}{|c|}{ (B) Melanoma } \\
\hline Patient & Cancer stage & Tissue sequencing & ddPCR & CPM \\
\hline 1 & 4 & BRAF WT & BRAF V600E & 61.6 \\
\hline 6 & $3 c$ & BRAF WT & BRAF WT & 0.88 \\
\hline 10 & 4 & $B R A F$ V600E & BRAF WT & 3091 \\
\hline 11 & 4 & $B R A F$ V $600 \mathrm{E}$ & BRAF WT & 1.6 \\
\hline 15 & 4 & $B R A F$ V $600 \mathrm{E}$ & BRAF WT & 1.76 \\
\hline 16 & 4 & $B R A F \mathrm{~V} 600 \mathrm{~K}$ & $B R A F \mathrm{~V} 600 \mathrm{~K}$ & 3.08 \\
\hline 19 & 4 & BRAF WT & BRAF WT & 0.9 \\
\hline 20 & 4 & $B R A F$ V600E & BRAF WT & 2251 \\
\hline 22 & $3 a$ & $B R A F \mathrm{~V} 600 \mathrm{~K}$ & $B R A F \mathrm{~V} 600 \mathrm{~K}$ & 16.5 \\
\hline 25 & 4 & $B R A F$ V $600 \mathrm{E}$ & $B R A F \mathrm{~V} 600 \mathrm{~K}$ & 6.26 \\
\hline 28 & 4 & $B R A F$ V $600 \mathrm{E}$ & No results & No results \\
\hline 29 & $3 c$ & $B R A F \mathrm{~V} 600 \mathrm{~K}$ & BRAF WT & 298.1 \\
\hline 30 & 4 & $B R A F$ V600E & BRAF V600K & 36.6 \\
\hline 33 & $1 a$ & $B R A F$ V600E & No results & No results \\
\hline 37 & 4 & $B R A F$ V $600 \mathrm{E}$ & $B R A F$ V $600 \mathrm{E}$ & 16.5 \\
\hline 38 & 1 & BRAF WT & BRAF WT & 0.44 \\
\hline 39 & 4 & BRAF WT & BRAF WT & 7.48 \\
\hline 40 & 4 & BRAF WT & BRAF WT & 0.55 \\
\hline
\end{tabular}

- $C P M=\frac{\text { copies of target } x \text { template volume }}{\text { volume analyzed of the duplicates }}$

AJCC: American Joint Committee on Cancer staging system; ddPCR: Droplet DigitalTM PCR; MUT: Mutation; WT: Wild-type.

munotherapy with nivolumab and ipilimumab. Our EVs analysis revealed a BRAF V600K mutation. One year after recruitment into our study, staging analysis showed subcutaneous, cerebral, intraabdominal, intradural and vertebral metastatic progress. Metastatic tissue from submammary skin was sequenced due to the advanced progress, verifying the mutation switch from BRAF V600E to BRAF V600K.

Patient 338 was diagnosed with sigmoid colon cancer and synchronous hepatic metastasis. Routine pathology analysis revealed KRAS wild-type in biopsied tissue from the rectum and sigmoid colon. The patient received FOLFOX/cetuximab combination therapy for 4 months, but presented with lung and hepatic metastasis 1 year after treatment. A blood sample was collected prior to resection of the liver metastasis. EV analysis detected subclones resistant to anti-EGFR therapy, harboring a KRAS G12D mutation.

The availability of EVs in various biological fluids like plasma and urine has prompted multiple studies on their use as potential biomarkers [22]. EVs are known as reservoirs of proteins, DNA and RNA, with a lipid bilayer membrane shielding intracellular cargo from extracellular conditions. Among their many roles, the vesicles are able to reflect the cellular condition at the time of release and pass on information from cell to cell [23]. To date, the mechanisms of sorting cellular components into EVs and for their specific uptake by designated cells are still unclear. In this study, the possibility of mutation profiling in EVs was explored in patients' plasma via Droplet Digital PCR. The MM and CRC patient cohorts consisted of 28 patients with metastatic disease and two patients in the early stage of the 
disease, all of whom underwent treatment prior to plasma sampling. EV-based mutational analysis was compared with tissue samples, demonstrating a concordance rate of 54 and $44 \%$ in the CRC and MM cohorts, respectively.

In previously published data from our group, KRAS and BRAF were profiled in cfDNA and compared with tissue samples using ddPCR. In a cohort of 65 CRC patients (stages I-IV), cfDNA was detected in $100 \%$ of the patients, with $55 \%$ correlation with tissue samples. Mutations were mainly detected in stage IV patients (and in one patient in stage II); two of ten patients had BRAF mutations and nine of 25 patients had mutations of KRAS [24]. The overall concordance rates demonstrated comparable utility of cfDNA (55\%) and EVs (54\%) analysis for tissue mutation detection in plasma of CRC patients. Three patients (patients 302,338 and 356 ) from the cohort were included in our EVs study to allow direct comparison of EVs and cfDNA. Interestingly, EVs may be more accurate in matching the gene status of the tumor when compared with cfDNA (while acknowledging that the comparison of cfDNA and EVs was limited with only three patients). The KRAS G12D tissue mutation of patient 302 was verified in both EVs and cfDNA. However, in patient 356 , EVs analysis displayed a KRAS G12V mutation, whereas cfDNA analysis only detected the wild-type. Disease progression in patient 338 correlated with EV-based ddPCR results, demonstrating a mutation switch; in contrast, only wild-type was detected in cfDNA. Our results corresponded with a report by Möhrman et al. [25], who analyzed EVs for BRAF V600 and KRAS G12/G13 via next-generation sequencing; cfDNA was analyzed with ddPCR and BEAMing digital PCR [25]. When compared with sequenced formalin-fixed, paraffin-embedded archival tissues, $95 \%$ of exosome samples matched the mutation status of the tissue. The concordance rate for cfDNA was comparable with EVs, with $90 \%$ for ddPCR and $92 \%$ for BEAMing digital PCR [25]. In another study of both liquid biopsy components, KRAS mutation detection was undertaken on exosome-derived DNA and cfDNA isolated from pancreatic ductal adenocarcinoma patients; when compared with tissue, exosome-derived DNA surpassed cfDNA in KRAS detection in metastatic patients (85 and 57.9\%, respectively) [9].

The discordant results can be explained by the heterogeneous landscape of the tumor and/or the different timepoints of plasma sample and tissue collection. In our study the blood samples were collected after patients received the allocated therapy; therefore, tissue sampling prior to treatment and blood collection post-treatment were separated by a median time difference of 6 months. Thus we explored whether EVs could provide information on how the treatment affects the disease. Möhrman et al. reported that the discrepancies in their analysis when comparing gene status in exo-nucleic acid and archival tissue samples could be due to time variation of plasma and tissue collection. They reported a much longer time difference (median 20 months) than in our study and a 5\% discordance rate between exo-nucleic acid and archival tissues [25]. Nevertheless, our study is not without limitations. Compared with other studies $[9,25,26]$, our cohort is relatively small, and the patients were treated with diverse therapies.

As it is difficult to obtain repeated tissue samples, it is beneficial to incorporate blood-based detection methods to assist in treatment decision making. Two cases in our patient cohort suggested that EV analysis could be used as part of treatment monitoring to detect resistance. The patients representing both $\mathrm{MM}$ and advanced $\mathrm{CRC}$ not only demonstrated a mutation switch, but also a significant disease progression.

Clinical trials demonstrated improved progression-free survival and overall survival in melanoma patients who received selective $B R A F$ (dabrafenib, vemurafenib) and MEK (trametinib) inhibitors compared with those receiving systemic therapy (e.g., dacarbazine, interferon or IL-2) [27]. However, unavoidably, subclonal activation during BRAF/MEK inhibition occurred at a median rate of 4-7 months post treatment [28]. This included a mutation shift, proliferation of subclones and eventually their detection by mutation testing. Van Allen et al. sequenced tissue samples originating from BRAF V600 mutant advanced melanoma patients and reported genetic alterations in the MAPK (ERK) signal transduction pathway in $51 \%$ of the patient cohort $[29,30]$. This was also noticed in patient 16 who demonstrated a significant progress 10 months after treatment pause, correlating with the presence of a mutation switch (BRAF V600E to BRAF V600K). Unlike sequenced tissue taken prior to BRAF/MEK inhibitor treatment, EV-based analysis demonstrated the BRAF V600K mutation. Interestingly, the EV-ddPCR results were reconfirmed by sequencing newly biopsied submammary metastatic skin tissue.

The anti-epidermal growth factor receptor (anti-EGFR) inhibitor cetuximab was approved as first-line single-agent therapy (or in combination with standard chemotherapy) for metastatic CRC patients who are intolerant to chemotherapy, either EGFR-expressing or RAS wild-type [31]. Molecular alterations have been reported in colorectal cancers that are RAS wild-type at baseline, eventually shifting the tumors to a mutated status resulting in a secondary resistance to anti-EGFR therapy [32]. We noticed a similar acquired resistance in patient 338 when we compared the EV status 1 year after FOLFOX/cetuximab combination treatment with that of primary tumor tissue biopsied prior to therapy. This disease progression was not detectable in our cfDNA study [24]. In cases from both the MM and CRC patient cohorts, EV-ddPCR indicated the mutation switch before known disease progression could be detected by either staging, routine $\mathrm{PCR}$ or tissue sequencing analysis.

We proved that EVs are able to provide a snapshot of disease evolution. Furthermore, EV-cDNA is able to detect mutation switches prior to clinically verified disease progression. Liquid biopsy is therefore a versatile addition to tissue biopsy for monitoring responses to therapy, a process that requires the possibility of repetitive real-time monitoring to understand the dynamic changes within the disease.

\section{Future perspective}

As a liquid biopsy component, EVs provide valuable information for real-time monitoring of disease and could be utilized as a cancer screening tool due to minimal invasiveness. Cancer is a heterogeneous disease and liquid biopsy could adequately reflect this information, unlike mutation analysis of tissue biopsy, which only provides information about the specific location where tissue was acquired. 


\section{Supplementary data}

To view the supplementary data that accompany this paper please visit the journal website at: www.futurescience.com/doi/suppl/10.2144/btn-2020-0028

\section{Acknowledgments}

The authors would like to thank $\mathrm{H}$ Heilmann for her excellent technical assistance with the whole-mount electron microscopy. They would also like to extend their gratitude to Berliner Krebsgesellschaft e.V. for the Ernst-von-Leyden Stipendium for recipient SA Yap.

\section{Financial \& competing interests disclosure}

The project was funded by German Cancer Consortium (DKTK), German Cancer Research Center (DKFZ) and German Cancer Aid. OncoTrack received research funding from the Innovative Medicines Initiative Joint Undertaking under grant agreement no. 115234, with financial contributions from EU's Seventh Framework Programme (FP7/2007-2013) and EFPIA companies. SA Yap was a recipient of the Ernst-von-Leyden Stipendium from the Berliner Krebsgesellschaft e.V. (October 2015-October 2017). U Keilholz has a possible conflict of interest with Amgen Inc., AstraZeneca, BMS, Boehringer Ingelheim, Glycotope GmbH, Innate Pharma, Lilly, Medlmmune, Merck Group, Serono, MSD/Merck and Co., Novartis, Pfizer, Roche/Genentech and Sirtex Medical. The authors have no other relevant affiliations or financial involvement with any organization or entity with a financial interest in or financial conflict with the subject matter or materials discussed in the manuscript apart from those disclosed.

No writing assistance was utilized in the production of this manuscript.

\section{Ethical conduct of research}

The authors state that all patients provided informed consent prior to blood donation and the study was approved by ethics committee.

\section{Open access}

This work is licensed under the Attribution-NonCommercial-NoDerivatives 4.0 Unported License. To view a copy of this license, visit http://creativecommons.org/licenses/by-nc-nd/4.0/

\section{References}

Papers of special note have been highlighted as: • of interest; $\bullet \bullet$ of considerable interest

1. Pantel K, Alix-Panabieres C. Liquid biopsy and minimal residual disease latest advances and implications for cure. Nat. Rev. Clin. Oncol. 16(7), 409-424 (2019).

- Demonstrates the importance of liquid biopsy.

2. Babayan A, Pantel K. Advances in liquid biopsy approaches for early detection and monitoring of cancer. Genome Med. 10(1), 21 (2018).

3. Alix-Panabières C, Pantel K. Circulating tumor cells: liquid biopsy of cancer. Clin. Chem. 59(1), 110-118 (2013).

4. Mader S, Pantel K. Liquid biopsy: current status and future perspectives. Oncol. Res. Treat. 40, 404-408 (2017)

5. Hoshino A, Costa-Silva B, Shen T-L et al. Tumour exosome integrins determine organotropic metastasis. Nature 527(7578), 329-335 (2015)

6. Théry C, Zitvogel L, Amigorena S. Exosomes: composition, biogenesis and function. Nat. Rev. Immunol. 2(8), 569-579 (2002).

7. Samanta S, Rajasingh S, Drosos N, Zhou Z, Dawn B, Rajasingh J. Exosomes: new molecular targets of diseases. Acta Pharmacol. Sin. 39(4), 501-513 (2018).

8. Skog J, Wurdinger T, van Rijn S et al. Glioblastoma microvesicles transport RNA and proteins that promote tumour growth and provide diagnostic biomarkers. Nat. Cell Biol. 10(12), 1470-1476 (2008)

9. Allenson K, Castillo J, San Lucas FA et al. High prevalence of mutant KRAS in circulating exosome-derived DNA from early-stage pancreatic cancer patients. Ann. Oncol. Off. J. Eur. Soc. Med. Oncol. 28(4), 741-747 (2017).

- Compared mutation detection in cell-free DNA (cfDNA) and exosomes-derived DNA.

10. Kahlert C, Melo SA, Protopopov A et al. Identification of double-stranded genomic DNA spanning all chromosomes with mutated KRAS and $p 53$ DNA in the serum exosomes of patients with pancreatic cancer. J. Biol. Chem. 289, 3869-3875 (2014).

11. Klump J, Phillipp U, Follo M et al. Extracellular vesicles or free circulating DNA: where to search for BRAF and cKIT mutations? Nanomedicine 14(3), 875-882 (2018).

12. San Lucas FA, Allenson K, Bernard V et al. Minimally invasive genomic and transcriptomic profiling of visceral cancers by next-generation sequencing of circulating exosomes. Ann. Oncol. Off. J. Eur. Soc. Med. Oncol. 27(4), 635-641 (2016).

13. Schütte M, Risch T, Abdavi-Azar N et al. Molecular dissection of colorectal cancer in pre-clinical models identifies biomarkers predicting sensitivity to EGFR inhibitors. Nat. Commun. 8 , 14262 (2017).

14. Ye J, Coulouris G, Zaretskaya I, Cutcutache I, Rozen S MT. Primer-BLAST: a tool to design target-specific primers for polymerase chain reaction. BMC Bioinformatics 13 , 134 (2012).

15. Bio-Rad. Droplet Digital application guide. http://www.bio-rad.com/webroot/web/pdf/lsr/literature/Bulletin_6407.pdf

16. Trajkovic K, Hsu C, Chiantia S et al. Ceramide triggers budding of exosome vesicles into multivesicular endosomes. Science 319(5867), 1244-1247 (2008).

17. Raposo G, Stoorvogel W. Extracellular vesicles: exosomes, microvesicles, and friends. J.Cell Biol. 200(4), 373-383 (2013).

18. Colombo M, Raposo G, Théry C. Biogenesis, secretion, and intercellular interactions of exosomes and other extracellular vesicles. Annu. Rev. Cell Dev. Biol. 30, 255-289 (2014).

19. György B, Szabó TG, Pásztói M et al. Membrane vesicles, current state-of-the-art: emerging role of extracellular vesicles. Cell. Mol. Life Sci. 68(16), 2667-2688 (2011).

20. Théry C, Ostrowski M, Segura E. Membrane vesicles as conveyors of immune responses. Nat. Rev. Immunol. 9(8), 581-593 (2009).

21. Théry C, Amigorena S, Raposo G, Clayton A. Isolation and characterization of exosomes from cell culture supernatants and biological fluids. Curr. Protoc. Cell Biol. Chapter 3: Unit 3.22 (2006).

-. Describes the standard isolation of EVs from cell lines and plasma

22. Kahlert C. Liquid biopsy: is there an advantage to analyzing circulating exosomal DNA compared to cfDNA or are they the same? Cancer Res. 79(10), 2462-2465 (2019).

23. Srivastava A, Amreddy N, Ahmed R et al. Exosome RNAs as biomarkers and targets for cancer therapy. Diagnostic Ther. Appl. Exosomes Cancer 2018, 129-159 (2018).

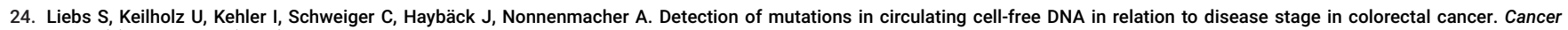
Med. 8(8), 3761-3769 (2019).

- cfDNA analysis of three patients from this study was compared with extracellular vesicles analysis in our study.

25. Möhrmann L, Huang HJ, Hong DS et al. Liquid biopsies using plasma exosomal nucleic acids and plasma cell-free DNA compared with clinical outcomes of patients with advanced cancers. Clin. Cancer Res. 24(1), 181-188 (2018). 
- Compared mutation load in nucleic acids from exosomes and cfDNA.

26. Kim Y, Shin S, Kim B, Lee K-A. Selecting short length nucleic acids localized in exosomes improves plasma EGFR mutation detection in NSCLC patients. Cancer Cell Int. 19, 251 (2019).

27. Menzies AM, Long GV, Murali R. Dabrafenib and its potential for the treatment of metastatic melanoma. Drug Des. Devel. Ther. 6, 391-405 (2012).

28. Johnson DB, Flaherty KT, Weber JS et al. Combined BRAF (Dabrafenib) and MEK inhibition (Trametinib) in patients with BRAFV600-mutant melanoma experiencing progression with single-agent BRAF inhibitor. J. Clin. Oncol. 32(33), 3697-3704 (2014).

29. Van Allen EM, Wagle N, Sucker A et al. The genetic landscape of clinical resistance to RAF inhibition in metastatic melanoma. Cancer Discov. 4(1), 94-109 (2014).

30. McCain J. The MAPK (ERK) pathway: investigational combinations for the treatment of BRAF-mutated metastatic melanoma. $P$ T 38(2), 96-108 (2013).

31. Van Cutsem E, Köhne $\mathrm{CH}$, Láng I et al. Cetuximab plus irinotecan, fluorouracil, and leucovorin as first-line treatment for metastatic colorectal cancer: updated analysis of overall survival according to tumor KRAS and BRAF mutation status. J. Clin. Oncol. 29(15), 2011-2019 (2011).

32. Parseghian CM, Loree JM, Morris VK et al. Anti-EGFR-resistant clones decay exponentially after progression: implications for anti-EGFR re-challenge. Ann. Oncol. Off. J. Eur. Soc. Med. Oncol. 30(2), 243-249 (2019).

- Demonstrated mutation switch due to anti-EGFR-resistant clones. 